\title{
Agency improves working memory and accelerates visual and attentional processing.
}

Rocio Loyola-Navarro',2,3, Cristóbal Moënne-Loccoz', Rodrigo C. Vergaras, Alexandre Hyafile, Francisco Aboitiz' ${ }^{7}$ Pedro E. Maldonado',2

1Departamento de Neurociencia, Facultad de Medicina, Universidad de Chile

${ }^{2}$ Biomedical Neuroscience Institute, BNI

${ }^{3}$ Departamento de Educación Diferencial, Facultad de Filosofía y Educación, Universidad Metropolitana de Ciencias de la Educación.

${ }^{4}$ Departamento de Ciencias de la Salud, Facultad de Medicina, Pontificia Universidad Católica de Chile.

${ }_{5}^{5}$ Departamento de Kinesiología, Facultad de Artes y Educación Física, Universidad Metropolitana de Ciencias de la Educación.

${ }^{6}$ Centre de Recerca Matemàtica

` Escuela de Medicina, Facultad de Medicina, Pontificia Universidad Católica de Chile.

Corresponding author:

Pedro E. Maldonado, Ph.D.

Departamento de Neurociencia

Facultad de Medicina

Universidad de Chile

Independencia 1027

Santiago, Chile

pedro@uchile.cl 


\begin{abstract}
Agency, understood as the ability of an organism to control stimuli onset, modulates perceptual and attentional functions. Since stimulus encoding is an essential component of working memory (WM), we conjectured that the perceptual process's agency would positively modulate WM. To corroborate this proposition, we tested twenty-five healthy subjects in a modified-Sternberg WM task under three stimuli presentation conditions: an unpredictable presentation of encoding stimulus, self-initiated presentation of the stimulus, and self-initiation presentation with random-delay stimulus onset. Concurrently, we recorded the subjects' electroencephalographic signals during WM encoding. We found that the self-initiation condition was associated with better WM accuracy, and earlier latencies of N100 and P200 evoked potential components representing visual and attentional processes, respectively. Our work demonstrates that agency enhances WM performance and accelerates early visual and attentional processes deployed during WM encoding. We also found that self-initiation presentation correlates with an increased attentional state compared to the other two conditions, suggesting a role for temporal stimuli predictability. Our study remarks on the relevance of agency in sensory and attentional processing for WM.
\end{abstract}

Keywords: EEG, embodied cognition, self-initiated stimuli, Sternberg task, temporal prediction 


\section{INTRODUCTION.}

While classic Working Memory (WM) paradigms require subjects to passively attend to task-relevant stimuli, that are presented without control of the participants, in everyday situations, stimuli relevant to WM tasks may appear to our sensory system as a consequence of the agent's movements, such as what occurs during eye movements or when we click on the mouse or the mobile screen to browse a web page. When the agent initiates the presentation of the stimuli, behavior and its underlying neural mechanisms are modulated in several cognitive processes such as perception (Blakemore et al. 1999) and attention (Nittono et al. 2003a; Nittono 2004; Nittono 2006; Waszak \& Herwig 2007; Kumar et al. 2015). This idea seems to be in line with the embodied cognition theory (O'Regan \& Noë 2001; Varela et al. 2016), which states that subjects' bodies, particularly their motor systems, influence cognition. Nevertheless, it remains unknown whether agency, understood as the control of stimuli onset by the agent (i.e., self-initiation), modulates WM encoding processing. Additionally, it has not been established if this influence is achieved by modulating sensory, attentional processes or later updating mechanisms of stimuli in memory. Revealing possible motor modulations of WM through self-initiation of stimuli may improve our understanding of the WM mechanism in real-life situations, hallmarking participants' role as agents of their own cognitive process.

What are the mechanisms deployed in agency that can possibly modulate WM encoding? Evidence in perceptual and attentional tasks have shown that the agents' self-initiation of stimuli modulates both sensory and attentional neural processing (Nittono et al. 2003b; Walsh \& Haggard 2008; Stenner et al. 2014; Concha-Miranda et al. 2019). This finding is relevant in WM's context since WM has been proved to rely on both perceptual (Teng \& Kravitz 2019; Zhang et al. 2019) and attentional processing (Postle 2006). Furthermore, attention is proposed as a critical component of WM, being responsible for the maintenance of items and the WM span limits (Cowan 2005). One proposed mechanism by which agency may influence perception is the 
modulation that movements exert on early sensory cortices (Schroeder et al. 2010; Stenner et al. 2015; Concha-Miranda et al. 2019). It has been reported in animal models that motor acts such as eye movements (specifically saccades) modulate primary sensory cortex firing rate and local field potentials (Ito et al. 2011; McFarland et al. 2015). In humans, a similar modulation has been described in the P100 Event Related Potential (ERP) component, which has been proposed as an index of the early visual response in the visual cortex (Luck et al. 1990; Clark et al. 1995; Di Russo et al. 2002; Sergent et al. 2005; Natale et al. 2006). Devia et al. (2017) reported that the amplitude of the P100 component is larger when the stimulus was fixated after a saccade compared to flashed stimuli (i.e., non-saccade-mediated fixation) in a free-viewing paradigm. They also described earlier latencies of the P100 component, suggesting that the visual cortex activates faster to visual stimuli when they are a consequence of a motor act, such as saccades, compared to visual stimuli that are passively sensed (Devia, Montefusco-Siegmund, Egaña, Maldonado 2017). The motor cortex activity, such as the supplementary motor area (SMA), has been proved to modulate the visual cortex in mice through anatomically direct connections (Leinweber et al. 2017). Although this has not been confirmed in humans, a hand movement such as a button press could also modulate the cortical visual processing through SMA-visual cortex connections. If this is true, self-initiation of the stimulus during WM encoding should yield a modulation in the early visual encoding indexes such as P100 and N100 ERP components. A similar mechanism could be operating in the modulation observed of self-initiation on attentional neural substrates. The evidence in attentional tasks have shown that agency increases the amplitude of both P300a and P300b components compared to passive externally paced stimuli (Nittono et al. 2003a; Nittono 2004; Nittono 2006). This evidence reveals that agency enhances attentional processing through both attentional capture (indexed by P300a) and updating mechanisms in memory (indexed by P300b). These findings suggest that motor commands could also modulate WM at a later stage of encoding. SMA is also connected to the cortical regions related to the attentional mechanisms 
involved in WM, namely posterior parietal cortex (PPC) (Reep et al. 1994; Wilber et al. 2015; Bozkurt et al. 2017) and dorsolateral prefrontal cortex (DLPFC) (Hasan et al. 2013; Bozkurt et al. 2017). Suppose SMA is modulating the activity of the PPC and DLPFC. In that case, a self-initiated encoding should be associated with a modulation of attention indexes during WM encoding, such as P300 (Polich 2007) and P200 (Dunn et al. 1998; Missonnier et al. 2004) ERPs components. There are no current reports about self-initiation modulation on the P200 component; however, earlier P200 latency correlates with better performance in WM tasks (Missonnier et al. 2007). As for motor actions modulating WM, self-initiation has been explored (McMillan et al. 2007; Holgado et al. 2019), but it has never been directly compared to passive conditions. There is, instead, behavioral evidence stating that task-unrelated voluntary motor acts worsen WM outcome (Gündüz et al. 2017), suggesting that motor sequence execution shares cognitive resources with WM. Nevertheless, whether this interference results from a sensory or/and an attentional neural modulation remains unknown.

Self-initiated tasks usually allow for temporal prediction of the stimuli appearance, since the movement that triggers the stimuli is finely coupled in time (i.e., time-locked) to the sensory consequence. Time-prediction, even if it does not occur in the context of self-initiation, refers to a neural mechanism in which the "processing and detection of events are facilitated by minimizing the uncertainty about when they are going to occur" (Arnal \& Giraud 2012). If the agency's modulatory effects rely on time-prediction only, then the jittering of the motor act and its sensory consequence should abolish those effects. Fine temporal coupling between motor acts and their sensory consequences have been depicted to be crucial to visual cortex development (Attinger et al. 2017) and important in perception (Blakemore et al. 1999) and attention (Morillon et al. 2014). However, studies indicate that movements that are made before and after stimuli presentation (hence, the stimuli are not an immediate consequence of the motor act) can also influence perception (Tomassini et al. 2015) and even long-term memory performance (Yebra et al. 2019). 
This evidence is suggestive of a possible motor-related modulation that is independent of temporal prediction. Yebra et al. (2019) indicated that the noradrenergic system's engagement mediates this motor-related improvement in long-term memory performance during encoding. Since the noradrenergic system massively modulates brain activity, it is possible to speculate an influence at both early sensory and attentional encoding processing levels.

Based on the literature reviewed above, we hypothesized that the agents' self-initiation of stimuli modulates WM encoding. This effect relies on the temporal modulation of visual, attentional and memory updating processing. To test this hypothesis, 25 healthy adults performed a modified Sternberg WM task (Sternberg 1966), with three different ways of deploying the stimuli, while EEG activity was recorded. The classic control WM paradigm (Passive (P) condition), consisting of the automatic presentation of the stimuli, was compared to two self-initiated conditions: an active coupled condition (AC) and an active decoupled condition (AD). If active self-initiated WM encoding improves WM through a temporal modulation of visual, attentional or memory updating processing, we should find: a) better performance in AC compared to $\mathrm{P}, \mathrm{b}$ ) an effect in encoding the ERPs markers in $\mathrm{AC}$ compared to $\mathrm{P}$, and $\mathrm{c}$ ) a relation between ERP modulation and performance. Moreover, to test if agency influence is based on temporal prediction, a decoupled encoding condition (AD) was designed. AD consisted of the presentation of the stimuli after a random delay (400-600 ms) after the button press, which reduces the temporal predictability of the stimuli onset without affecting the sense of agency (Wen 2019). In case the modulatory effects of agency rely on time-prediction only, we should find: a) no significant differences in performance and ERP components between $\mathrm{AD}$ and $\mathrm{P}$ conditions, since both conditions lack of precise timeprediction of stimuli onset, and b) statistically significant difference in performance and ERP components between $A C$ and $A D$. Our results show an effect of task conditions on both performance and ERP components, with AC yielding better performance and earlier latencies of ERP components compared to both AD and P conditions. Moreover, AD condition also presents 
better performance and earlier latencies of ERP components compared to P condition, suggesting that agency does not rely on the temporal coupling between action and its sensory consequence only.

\section{MATERIALS AND METHODS}

\section{Participants}

Twenty-six healthy adults (13 females; mean age 23.1 y.o.; range 18-31 y.o.) volunteered and were tested. All the participants were under- or postgraduate students of the Universidad de Chile with non-current or history of neurological, psychiatric, systemic disease, and normal or corrected-to-normal vision. This information was corroborated by anamnesis and the application of the MoCA test (Nasreddine et al. 2005). In the MoCA test, participants had to score $\geq 26$ points to be included. As for anamnesis, the exclusion criteria were: a) Cranioencephalic trauma; b) Usage of illegal drugs during the last three months; c) Uncompensated systemic disease (metabolic disease or epilepsy); d) Usage of one or more of these drugs: benzodiazepines, anticonvulsants, metilfenidate, modafinil; and e) Diagnosis of depression or adult attentional deficit disorder. Informed consent was previously read and signed by the participants. One of the 26 participants was excluded because of luminance differences during testing. Consent for the current study was approved by the Ethical Committee in Human's Research of the Medicine Faculty of the Universidad de Chile.

\section{Task}

Participants engaged in a modified Sternberg working memory task (Sternberg 1966), with three encoding conditions while recording EEG activity. The three conditions differed only in how the stimuli were triggered (whether controlled by the agent or externally) while sharing the same time course from stimuli presentation until the participants' answers. Conditions corresponded to 
an Active Coupled condition (AC), an Active Decoupled condition (AD) and a Passive condition (P). Details of the task scheme are shown in Figure 1.

In all the three conditions, each trial began with an eye-tracking-based drift correction to ensure that participants' eyes positions remain similar in every trial. After drift-correction, a first fixation cross marked the beginning of each trial. The stimuli array (S1) lasted 250 milliseconds (ms). After $\mathrm{S} 1$ disappeared, only the central fixation cross remained for a random time between 2000 and 2500 ms (maintaining). Subsequently, the probe stimulus appeared for 800 ms, followed by the final fixation cross, which lasted $1500 \mathrm{~ms}$ (retrieval). Subjects had all the retrieval time to respond $(800 \mathrm{~ms}+1500 \mathrm{~ms}=2300 \mathrm{~ms})$. If the probe was present in the $\mathrm{S} 1$ array, participants had to press the joystick's right back button with their right index finger. Conversely, if the probe was absent from set S1, participants had to press the left-back button with their left index finger.

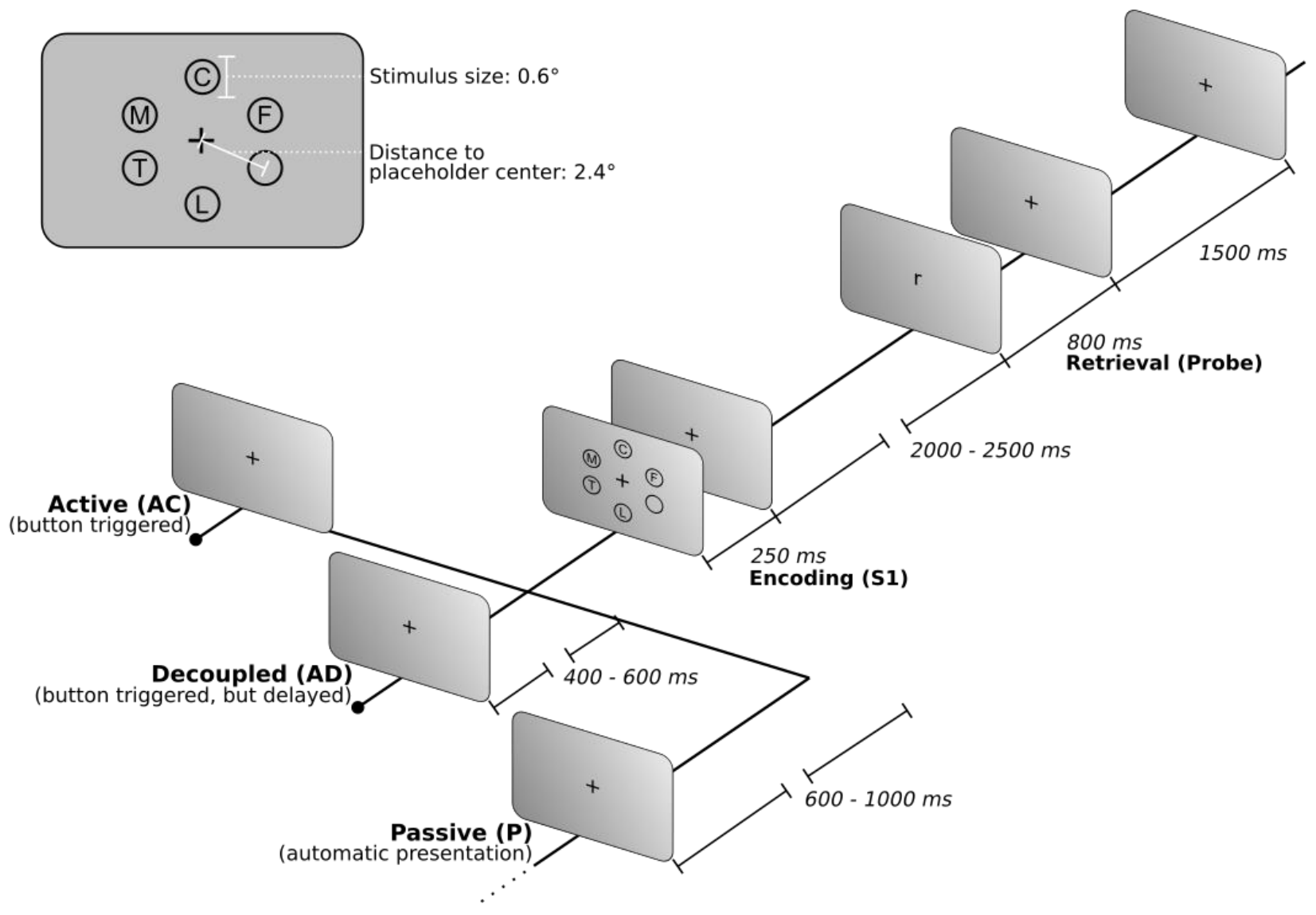


Figure 1. Schematic presentation of the WM task showing the three experimental conditions. Each condition varies only on the pre-encoding stage. Active coupled condition (AC) starts with a button press, and the stimuli array (S1) is displayed immediately. Decoupled condition (AD) also starts with a button press, but the $\mathrm{S} 1$ is delayed by a random time between 400 and 600 ms. Finally, Passive condition (P) follows an automatic presentation of $\mathrm{S} 1$ at a random time between 600 and $1000 \mathrm{~ms}$ following fixation cross onset. The inset shows an example of the $\mathrm{S} 1$ array and its visual disposition. ( ${ }^{\circ}$ visual degrees; ms: milliseconds).

As stated before, to determine if the agents' self-initiation of WM modulates WM, the onset of the $\mathrm{S} 1$ set was manipulated (i.e., the pre-encoding stage), generating three task conditions: AC, $A D$, and $P$. In the $A C$ condition, participants were required to press a frontal button of the joystick (with either of their thumbs). At the same time, the first fixation cross was present. Immediately after the button press, the $S 1$ array appeared on the screen for $250 \mathrm{~ms}$. The AD condition was very similar: participants were also required to press a frontal button. At the same time, the first fixation cross was present, but in this condition, S1 appeared with a random delay (400-600 ms) after the button press. This time-lapse does not disturb the sense of agency of individuals (Wen 2019). The P condition corresponds to the classic automatic presentation of the stimuli, which appear after a random fixation time of $600-1000 \mathrm{~ms}$.

Each condition was presented in separate blocks of 100 trials. Each participant executed all the three blocks, accomplishing a total of 300 trials. The presentation order of the blocks was pseudo-randomized per participant. Instructions were given separately for each condition. Participants were not told about the specific difference between $A C$ and $A D$ conditions to not bias their answers. For participants 12 to 25 , at the end of each block, they were asked to determine the task's difficulty. To do so, they rated their global sensation of how secure they made their answers in that particular block (hereafter "confidence"). A scale of confidence ranging from 1 to 7 was used, with 1 corresponding to "not sure of my answers," 4 being neutral, and 7 corresponding to "very sure of my answers." The experimental paradigm was designed in 
Experiment Builder (SR Research Ltd., Mississauga, Ontario, Canada) and executed in Eyelink 1000 (SR Research Ltd., Mississauga, Ontario, Canada).

\section{Materials}

The stimuli were presented in a flat Viewsonic 27 inches screen $(23.54 \times 13.24$ inches; $1920 \times 1080$ pixels of resolution). Subjects were seated at a $72 \mathrm{~cm}$ distance from the screen. Therefore, 40.35 pixels equals 1 visual degree $\left(^{\circ}\right)$. Images were $45.1^{\circ}$ wide and $26.3^{\circ}$ high. Subjects were required to maintain their chin on a chinrest during the whole experiment to reduce head movements and maintain a stable distance between the eyes and the screen. Fatigue blinks and eye positions were monitored with an Eyelink 1000 (SR Research Ltd., Mississauga, Ontario, Canada) eye-tracking system.

The background was set to gray (127/127/127 RGB) during the whole session to avoid luminance changes. All the stimuli ( $\mathrm{S} 1$ and probe stimulus) were black consonants. $\mathrm{S} 1$ consisted of 5 capital consonants (except X, Y) presented simultaneously in a circular array surrounding a fixation cross ( Fig. 1). The fixation cross appeared at the center of the screen (at pixel coordinates $960,540)$. Stimuli positions were demarcated by six circles as placeholders (based on Proksch \& Bavelier 2002; Green \& Bavelier 2003). Therefore, one circle was left empty in every trial. Each of the six circle positions had the same probability of being empty. As for the stimuli's size, consonants were set to a height of $0.6^{\circ}$, while the placeholders' circles had $1^{\circ}$ diameter (based on Proksch \& Bavelier 2002; Green \& Bavelier 2003). Placeholders centroids were set at a $2.4^{\circ}$ distance from the central fixation cross. Since the parafoveal region has a size of $5.2^{\circ}$ (Kolb 1995), this disposition ensured that the stimuli inside the placeholders fit into the parafoveal region. Three hundred S1 sets were created, one for each trial. Each S1 set consisted of five unrepeated consonants chosen randomly by "rand" function in Matlab@. Consonants "B", "V", and "W" never appeared in the same set S1; the same occurred with "M" and "N". This was done to avoid acoustical or visual associations. As for the probe stimulus, it consisted of a unique consonant 
(excluding "x" or "y") presented in the center of the screen (coordinates 962,540). The presence or absence of the probe stimuli on the $\mathrm{S} 1$ set had the same probability (50\%). Responses were made by pressing a button with either left (probe is not present on S1) or right (probe is present in S1) index finger. A joystick, model Microsoft SideWinder, was used to record the response.

\section{Behavioral data analysis}

To assess the effects of agents' self-initiation on WM, the encoding condition's effect on accuracy and reaction times was analyzed by performing two One-way-repeated-Analysis of Variance (ANOVA) and pertinent post hoc tests when needed. Accuracy was defined as the proportion of correct answers (True Positives and True Negatives) for each participant. Reaction time (RT) corresponded to the time (in ms) elapsed since the appearance of S2 until the answer made with the button press. The effects of the encoding condition on the two dependent variables were analyzed using two One-way-repeated ANOVA. Post hoc tests for each variable with significant effects were computed using a paired-t-test corrected by the Holm-Sidak method's multiple comparisons. These parametric tests were chosen according to results of normality distribution (assessed by Shapiro-Wilk test) and of equality of variances (assessed by Levene test) of both accuracy and RT (See Table 1).

The effects of other variables that could influence WM behavior were tested next. Thus, a binomial General Linear Mixed Model (GLMM) was performed (Moscatelli et al. 2012). Trial accuracy was set as the dependent variable and participant as a random effect. GLMM included the following variables as fixed effects: encoding condition, task learning, block position, and prestimuli time. Task learning was defined as the change in the probability of making a correct response due to the increasing trial number (from 1 to 100). The block position was a categorical variable, and its value could be first, second, or third block. Finally, pre-stimuli time was analyzed in two different ways (and therefore, in two separate models): a) a total pre-stimuli time was 
defined as the time elapsed from the first fixation cross onset until the onset of the $S 1$ array, b) uncontrolled pre-stimuli time, defined as the time previous to stimuli onset that the subjects could not control. In the passive condition, both the total and uncontrolled pre-stimuli times were the same: the first fixation cross duration. In the $A D$ condition, uncontrolled pre-stimuli time was the delay time between the button press and the $S 1$ set appearance; total pre-stimuli time was the delay time plus the time the participant took to press the frontal button while the first fixation cross was present. Finally, in the AC condition, the uncontrolled pre-stimuli time is 0 , while the total prestimuli time was the time the participant took to press the frontal button. All the four independent variables were standardized using z-score transformation. The Akaike information criterion (AIC) was applied to select the best model.

\section{Electroencephalography and Signal pre-processing.}

Electroencephalographic (EEG) activity was recorded at a $2048 \mathrm{~Hz}$ sample rate using a BioSemi Inc. amplifier of 32 active scalp electrodes and eight external electrodes to record eye movements. Common Mode Sense (CMS) and Driven Right Leg (DRL) electrodes were used as ground electrodes. Head caps were utilized to hold electrodes according to the $10 / 10$ system (Jurcak et al. 2007). Eight external electrodes were set: one for each mastoid and six to record the eye movements (EOG): three around the right orbit and three around the left orbit.

Continuous EEG signal was re-referenced offline to the average activity of the 32 electrodes. Signal was then filtered using a FIR symmetric passband filter between 0.5 and $40 \mathrm{~Hz}$ with a linear phase. Its design is firwin with a Hamming window (acausal, zero-phase delay, and one-pass forward). The size of the filter was 6.6 seconds. The transition bandwidth of the filter was $0.5 \mathrm{~Hz}$ in the lower frequency limit and $10 \mathrm{~Hz}$ in the upper-frequency limit. The passband ripple of the filter was $0.0194 \mathrm{~dB}$ and the stopband attenuation was $53 \mathrm{~dB}$. 
Noisy channels were eliminated by visual inspection and then interpolated using spherical splines. After that, an Independent Component Analysis (ICA) was performed to determine and eliminate components related to blinks and eye movements. Segments containing muscle artifacts and other artifacts unrelated to blinks were eliminated automatically through a $500 \mu \mathrm{V}$ peak-topeak rejection threshold.

\section{Event-Related Potential (ERP) Components calculation}

Continuous Signal was divided into epochs centered on the stimuli's appearance. The epoch was set to $500 \mathrm{~ms}$ before and $1000 \mathrm{~ms}$ after the stimuli appearance. Noisy epochs were rejected using a $250 \mu \mathrm{V}$ peak-to-peak threshold. We explored ERP components related to those processes to analyze the influence of agency in early visual, attentional, and memory updating processing. Components of interest were N100-like, P200-like, and P300-like (hereafter, N1, P2, P3). We used electrode $\mathrm{Oz}$ to calculate P1 and N1 components, Fz to calculate the P2 component, and Pz to obtain the P3 component. All ERPs' components were computed by averaging correct cleaned trials. Amplitudes and latencies of each ERP component were assessed. Amplitude was calculated using peak-to-peak values. We used this approach since a component near $0 \mathrm{~ms}$ appeared in AC condition only, which could influence the peak amplitude values in the AC condition. The peak-to-peak method consisted of calculating the component's peak amplitude in a certain electrode and time-window, then calculating a reference value and subtracting both the values. To determine each component's peak value, the latency of that component was first calculated from the grand-average (average of the ERP across all 25 subjects). The peak amplitude and latency in each subject were then calculated in a 100 ms window around the grandaverage latency. The reference value for the $\mathrm{N} 1$ component corresponded to $\mathrm{P} 1$, while the reference values for P2 and P3 were defined as the negative peak appearing immediately after either P2 or P3. This data was calculated using only 24 of the 25 subjects since one of the 
participants was discarded because he had no reliable ERP responses. EEG data were preprocessed and averaged using MNE-Python (Gramfort 2013).

\section{Event-Related Potential (ERP) statistical analysis}

To test if there is an effect of the encoding condition over amplitude and latency, KruskalWallis, or ANOVA tests (one per ERP component) were performed, designed as CONDITIONSXLATENCIES and CONDITIONSXAMPLITUDES. The Rank-sum Wilcoxon or t-test corrected by Holm-Sidak was performed as a Post Hoc test. These tests were selected based on the distribution of the data (amplitude and latency) using the Shapiro-Wilk and Levene tests, respectively. To assess which electrophysiological activity distinguishes better among experimental conditions, a conditional Classification Tree (CART model) was used. CART models allow us to classify or estimate phenomena with discrete changes (Hothorn, Hornik, Van De Wiel, et al. 2006; Hothorn, Hornik, \& Zeileis 2006; Strobl et al. 2009). For this particular CART model, the Monte Carlo Method was selected, with 1000 resampling to estimate tree splits' significance using an alpha of 0.05 . Additionally, to avoid overfitting the model, the amount of observation per leaf was limited to $20 \%$ of the total observations. The partitioning variables used to characterize the experimental conditions were accuracy, RT, and the amplitudes and latencies of P1N1, P2, and P3 components.

To test if the relationship between the better electrophysiological variable and accuracy is dependent on the task condition, a Linear Mixed Model was performed. The model included accuracy as a dependent variable, the best electrophysiological variable (yielded by the CART model) as a fixed effect, and the encoding conditions as a random effect variable.

All computations were performed using RStudio (Rstudio Team 2016) for statistical computing. Libraries used were EZ (v4.4-0) (Lawrence 2016), car (v3.0-2) (Fox \& Weisberg 2014), party (v1.3-5) (Hothorn et al. 2015), and Ime4 (v1.1-21) (Bates et al. 2015). 


\section{RESULTS}

The current work hypothesized that the self-initiated WM stimulus presentation improves WM performance through the temporal modulation of visual, attentional, and memory updating processing. To evaluate how the agent's self-initiation of stimuli would affect WM encoding, 25 participants performed a Sternberg working memory task, manipulating the stimuli's onset. In the Active Coupled (AC) condition, the stimuli were presented immediately after the participants pressed a button. In the Active Decoupled condition (AD), stimuli were presented with a delay of 400 to 600 ms after the button press, which allowed to test whether possible effects of self-initiation are tied to temporal precision of stimulus onset. Finally, these two self-initiated conditions were contrasted with the passive presentation of the stimuli $(P)$, where stimuli were automatically presented between 600-1000 ms after the first fixation onset. Each encoding condition consisted of one block of 100 trials so that each subject executed three separate blocks (one per encoding condition) of 100 trials each (See Materials and Methods).

Two approaches tested the hypothesis proposed: i) Is behavior modulated by these three experimental conditions? and ii) Does self-initiation modulate early visual, attentional, and memory updating processing during encoding?

\section{Agents' self-initiation of the stimuli enhance WM performance}

To explore whether the agency modulates WM mechanisms, we tested the effect of Encoding Conditions ( $A C, A D$, and $P$ ) on performance (RT and accuracy). Table 1 shows averages, standard deviations, and results of Shapiro-Wilk and Levene tests for reaction times (RT) and accuracy, specified per encoding condition. The ANOVA results reveal a main effect of Encoding condition on accuracy $\left(F(2,48)=25.67, p=2.6 e-08 ; \eta^{2} G=0.2\right)$. No Encoding Condition 
effects were found on $\mathrm{RT}(\mathrm{F}(2,48)=1.06, \mathrm{p}=0.35)$ ( $\underline{\text { Fig. } 2})$. These results reveal that agency modulates accuracy without affecting RT.

Table 1. Values of the dependent and independent variables per encoding condition

\begin{tabular}{|c|c|c|c|c|}
\hline & $\begin{array}{l}\text { Normality and } \\
\text { Variance tests }\end{array}$ & $\begin{array}{l}\text { Active Coupled } \\
\qquad \text { (AC) }\end{array}$ & $\begin{array}{l}\text { Active Decoupled } \\
\text { (AD) }\end{array}$ & $\begin{array}{c}\text { Passive } \\
\text { (P) }\end{array}$ \\
\hline Response & Shap-Wilk: $\mathrm{p}=0.32$ & $\bar{X}: 1096.22$ & $\bar{X}: 1057.18$ & $\bar{X}: 1099.88$ \\
\hline Times (ms) & Levene: $\mathrm{p}=0.69$ & SD: 196.20 & $S D: 190.6$ & $S D: 210.28$ \\
\hline \multirow[t]{2}{*}{ Accuracy } & Shap-Wilk: $p=0.83$ & $\bar{X}: 0.84$ & $\bar{X}: 0.78$ & $\bar{X}: 0.74$ \\
\hline & Levene: $\mathrm{p}=0.59$ & SD: 0.07 & SD: 0.08 & SD: 0.07 \\
\hline N1 Amplitude & Shap-Wilk: $p=0.03$ & Med: 9.953 & Med: 10.627 & Med: 8.694 \\
\hline$(\mu \mathrm{V})$ & Levene: $p=0.57$ & SD: 3.76 & SD: 3.65 & SD: 4.1 \\
\hline N1 Latency & Shap-Wilk: $p=0.87$ & Med: 172.60 & Med: 185.05 & Med:188.72 \\
\hline (ms) & Levene: $\mathrm{p}=0.26$ & SD: 10.08 & SD: 6.84 & SD: 9.19 \\
\hline P2 Amplitude & Shap-Wilk: $p=8 e-5$ & Med: 6.121 & Med: 6.099 & Med: 6.18 \\
\hline$(\mu \mathrm{V})$ & Levene: $\mathrm{p}=0.2$ & SD: 3.59 & SD: 1.93 & SD: 2.33 \\
\hline P2 Latency & Shap-Wilk: $\mathrm{p}=0.8$ & Med: 169.92 & Med: 184.81 & Med:190.67 \\
\hline (ms) & Levene: $\mathrm{p}=0.78$ & SD: 10.14 & SD: 10.02 & SD: 8.09 \\
\hline P3 Amplitude & Shap-Wilk: $p=1 e-5$ & Med: 1.836 & Med: 1.879 & Med: 2.093 \\
\hline$(\mu \mathrm{V})$ & Levene: $\mathrm{p}=0.69$ & SD: 1.76 & SD: 1.88 & SD: 1.53 \\
\hline P3 Latency & Shap-Wilk: $p=0.04$ & Med: 25.439 & Med: 330.81 & Med:323.48 \\
\hline (ms) & Levene: $\mathrm{p}=0.66$ & SD: 47.86 & SD: 46.31 & SD: 60.66 \\
\hline
\end{tabular}

To assess if agents' self-initiation enhances WM performance, we executed a post hoc test, analyzing the accuracy difference between $\mathrm{AC}$ (mean $=0.84 \pm 0.07$ ) and $\mathrm{P}$ conditions (mean $=0.74 \pm 0.07)$. Paired t-tests corrected by the Holm-Sidak method show that self-initiation presents 
better accuracy than the $P$ condition $(t=8.214 ; p=5.9 e-08)$. To further investigate if the timing between motor acts and its sensory consequences is relevant to the agency effect, a post hoc test analyzing the differences between $A C$ and $A D$ conditions (mean $=0.78 \pm 0.08$ ) was performed. Paired t-tests corrected by Holm-Sidak method also confirm better accuracy in AC compared to $A D$ condition $(t=3.259 ; p=0.0033)$. Noteworthy, when subjects engage in a passive WM task, their accuracy is poorer when compared to both active conditions ( $A C$ compared to $P: t=8.214$; $p=5.9 e-08, A D$ compared to $P: t=3.565 ; p=0.0031)$. This indicates the impact of agency on WM performance, which seems to be partially linked to action/stimuli coupling. A remarkable point is that these accuracy effects do not seem to be explained by differences in the perceived difficulty of the task since there are no significant differences in their reported confidence, rated by the participant at the end of each condition block $\left(\chi^{2}=1.472 ; p=0.478\right)$ (Fig. $\left.2 \mathrm{C}\right)$.

We then explored the impact of other cognitive factors on performance. The other factors tested were task learning (i.e., the index of trial within the block), block position, total pre-stimuli time (i.e., the time between the first fixation onset and the stimuli onset), and uncontrolled prestimuli time (i.e., the time between the first fixation onset and stimuli onset in the passive condition and the time between the button press and the stimuli onset in the AD and AC condition) (for further details, see Behavioral data analysis). To choose which pre-stimuli time has a greater effect on performance, we performed two binomial General Linear Mixed Models (GLMM). Both models just varied in which pre-stimuli time was used as fixed effects, including trial accuracy as the dependent variable and subjects as a random effect. The first model's fixed effects were encoding condition, task learning, block position, and total pre-stimuli time. The second model's fixed effects were encoding condition, task learning, block position, and uncontrolled pre-stimuli time. According to the Akaike Information Criterion (AIC), the second model is significantly better than the first model $(\triangle \mathrm{AIC}=7,2)$. The best model results show a significant effect in three of the independent variables assessed: task learning $(\boldsymbol{\beta}=0.20 \pm 0.09, z=2.25, p=0.024)$, block position 
$(\boldsymbol{\beta}=0.139 \pm 0.04, z=3.914, p=9.1 e-05)$, and the encoding conditions $(A D$ condition: $\boldsymbol{\beta}=-$ $0.779 \pm 0.195, z=-3.992, p=6.55 e-05 ; P$ condition: $\boldsymbol{\beta}=-0.976 \pm 0.317, z=-3.077, p=0.002)$ (Fig. 2D). Although the second model including the uncontrolled pre-stimuli time was better than the first one, results yield just a non-significant trend for the uncontrolled pre-stimuli time $(\boldsymbol{\beta}=$ $0.263 \pm 0.136, z=1.923, p=0.054)$. This implies that the probability of making a correct response is not modulated by the random time that the stimuli takes to appear in the AD and P conditions. We did not find significant interaction between task learning and block position $(\boldsymbol{\beta}=-0.065 \pm 0.03$, $z=-1.840, p=0.06$ ), nor interaction between task learning and tasks (Task Learning and AD condition: $\boldsymbol{\beta}=0.114 \pm 0.07, z=1.55, p=0.120 ;$ Task Learning and $P$ condition: $\boldsymbol{\beta}=-0.080 \pm 0.719$, $z=-1.114, p=0.26)$. These GLMM results corroborate the previous ANOVA analysis, indicating a significant decrease in the probability of making a correct response when subjects are engaged in the $A D$ or the $P$ conditions compared to $A C$ condition. The probability of making a correct response falls 0.77 log-odds points in the AD condition, and the P condition's 1.14 log-odds point. Alongside the task learning effect, results show that the probability of making a correct response rises in 0.2 log-odds points with each trial (from 1 to 100) within one block. Results also yield no interaction between task learning and encoding conditions, showing that task learning has the same effect on all three conditions. Likewise, the model reveals that the probability of answering correctly increases in 0.13 log odds with the block position. Like in task learning, the effect of the block position is also independent of the encoding condition. Altogether, GLMM yields other factors such as task learning and block position that influence performance alongside the task conditions effects.

In summary, behavioral analyses show that coupled self-initiation of the stimuli in a WM task increases the probability of performing correctly, even though other timings and learning factors also modulate this probability. 

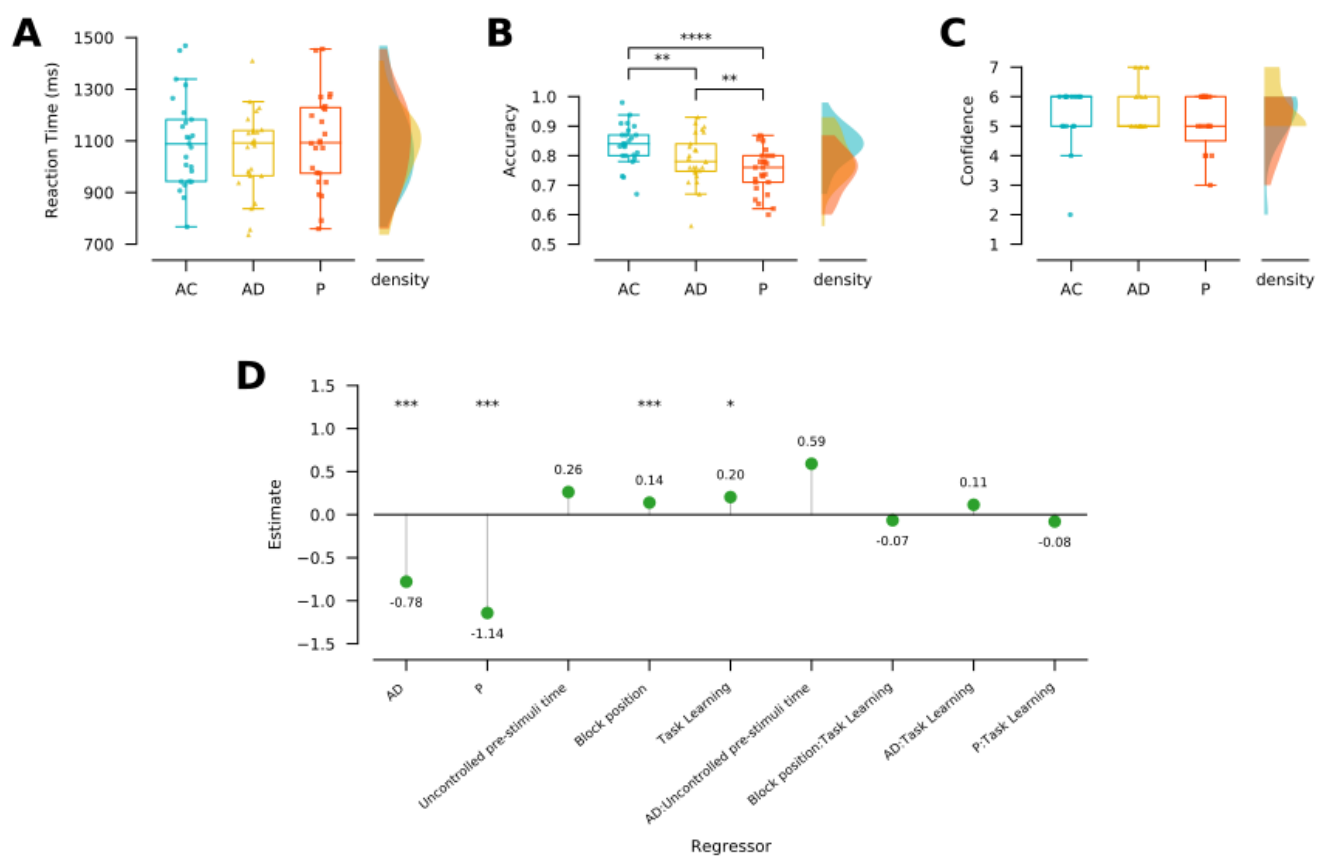

Figure 2. Agents' self-initiation of the stimuli enhances WM performance. A. Reaction times per encoding condition. Left panel, box plot shows the reaction times in milliseconds (ms) per condition (AC: light blue; AD: yellow; P: orange). Right panel, density plot shows the distributions of the reaction times per encoding condition ( $n=24)$. Dots in box plots represent the value for each participant. Upper limit of the box $=75^{\text {th }}$ percentile; lower limit of the box $=25^{\text {th }}$ percentile; upper whisker $=$ upper limit value; lower whisker $=$ lower limit value; outlier values are shown outside the whiskers. B. Similar to A, but for accuracy $(n=24)$. C. Similar to $A$, but for Confidence ratings $(n=15)$. D. Effects of variables (regressor) over the estimated value of making a correct answer. Colon (:) indicates interactions between parameters. $(*: p \leq 0.05 ; * *: p \leq 0.01$; $\star * *: p \leq 0.001 ; * * \star *: p \leq 0.0001)$.

\section{Agents' self-initiation of the stimuli accelerate N1 and P2 latencies during WM encoding}

We then assessed whether self-initiation of the stimuli impacts visual, attentional, and memory updating processing during WM encoding. To do so, the amplitudes and latencies of related ERP indexes were analyzed: N100-like component, proposed as an index of early visual discrimination related to the visual cortex (Vogel \& Luck 2000); P200-like component, which is thought to reflect attentional processing of stimuli in WM (Dunn et al. 1998); and P300-like 
component, a marker proposed as an index of mental revision of the stimuli (Polich 2007). The ERP's grand averages for correct trials are shown in Figure 3, specified per encoding conditions.

The ANOVA results reveal a significant effect of task conditions on the latency of the N1 $\left(F(2,46)=28.88, p=7.48 e-09 ; \eta^{2} G=0.37\right)$ and the $P 2$ components $(F(2,46)=46.701, p=8.418 e-$ $\left.12, \eta^{2} G=0.449\right)$. No statistically significant effect of encoding conditions were found on P3 latency $\left(\chi^{2}=0.242, p=0.886\right)$ and on amplitude of all the components analyzed $(N 1: F(2,46)=0.037, p$ $\left.=0.963 ; \mathrm{P} 2: \chi^{2}=0.089, \mathrm{p}=0.956 ; \mathrm{P} 3: \chi^{2}=0.328, \mathrm{p}=0.848\right)$. These results suggest a modulatory effect of task conditions on sensory and attentional processes, but not on memory updating mechanisms.

Two post hoc tests were performed, assessing the difference of $\mathrm{N} 1$ and $\mathrm{P} 2$ latencies between $\mathrm{AC}$ and $\mathrm{P}$ conditions. Paired t-tests corrected by the Holm-Sidak method show that subjects have earlier latencies in AC compared to $\mathrm{P}$ condition, both in $\mathrm{N1}$ ( $\mathrm{t}=-8.204 ; \mathrm{p}=8.3 \mathrm{e}-08)$ and P2 $(t=-9.431 ; p=6.8 e-09)$. Passive condition, on the other hand, yields the latest latencies in both $\mathrm{N} 1(\mathrm{AC} / \mathrm{P}: \mathrm{t}=-8.204 ; \mathrm{p}=8.3 \mathrm{e}-08 ; \mathrm{AD} / \mathrm{P}: \mathrm{t}=-3.009 ; \mathrm{p}=0.006)$ and $\mathrm{P} 2(\mathrm{AC} / \mathrm{P}: \mathrm{t}=-9.431$; $p=6.8 \mathrm{e}-09 ; A D / P: t=-2.1 ; p=0.047)$ components. These results suggest an effect of self-initiation of the stimuli on sensory and attentional processing.

To further examine if the temporal coupling between motor acts and its sensory consequences is relevant to the agency effect, two post hoc tests we performed, analyzing the N1 and P2 latency differences between AC and AD conditions. Paired t-tests corrected by the HolmSidak method also confirm earlier latencies of AC both in $N 1(t=-4.224 ; p=0.0006)$ and P2 $(t=$ $-6.461 ; p=p=2.7 e-06)$. As in behavioral analysis, these ERP results suggest that the agency effect is partially linked to the temporal coupling between action and its sensory consequence.

Note that all the three electrodes plotted (Oz, Fz, and Pz) show a component around stimuli presentations, specifically in AC condition. We interpret this component as a neural marker of the motor system activity related to the button press. Consistently, this component is also present in 
AD condition when ERPs are locked to the button press instead of stimuli presentation (See Figure S1).

In summary, the abovementioned results support that coupled self-initiation modulates the temporal domain of the neural mechanisms underlying the visual discrimination process (N1 component) and the attentional processes engaged during encoding in WM (P2 component), but there is no statistically significant effect on memory updating mechanisms (P3 component). Moreover, no effects on amplitudes were observed. Results also show that delays between movements and their sensory consequences (as reflected in AD condition) do not yield the same effect on encoding processing. Nevertheless, on comparing AD to passive condition, the latencies of $\mathrm{N} 1$ and $\mathrm{P} 2$ appear earlier. 


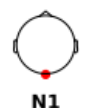

A

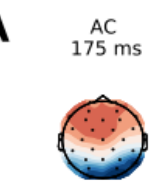

$\mathrm{AD}$
$185 \mathrm{~ms}$

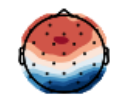

$\mathrm{Oz}$

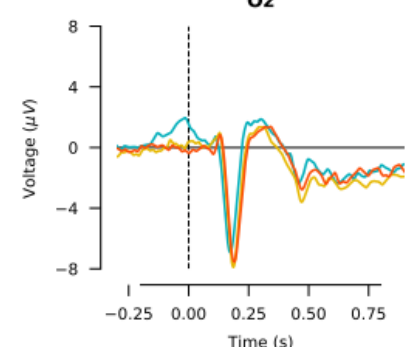

B
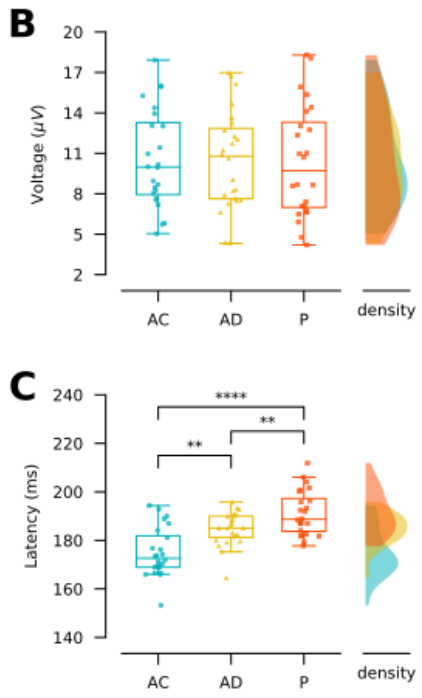

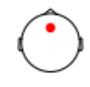

P2

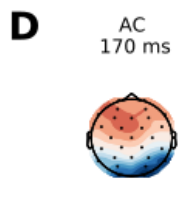

$\begin{array}{cc}\mathrm{AD} & \mathrm{P} \\ 185 \mathrm{~ms} & 190 \mathrm{~ms}\end{array}$
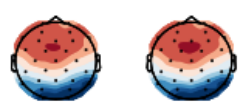

Fz
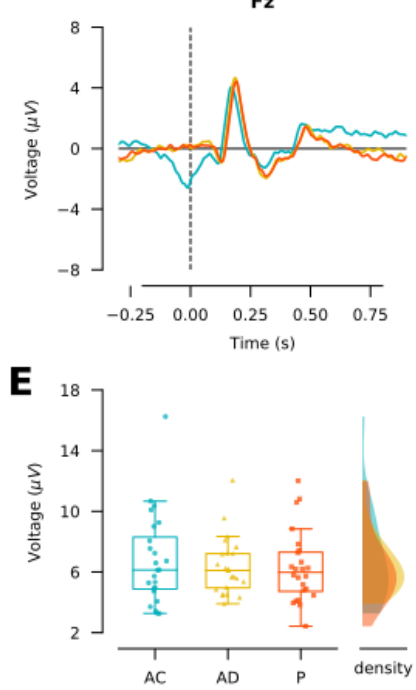

H

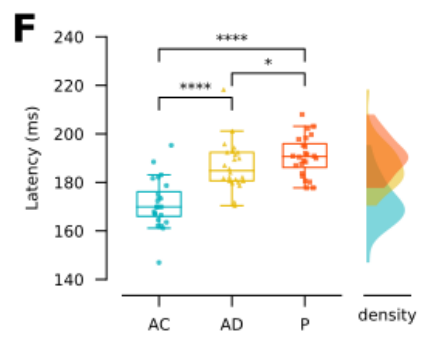

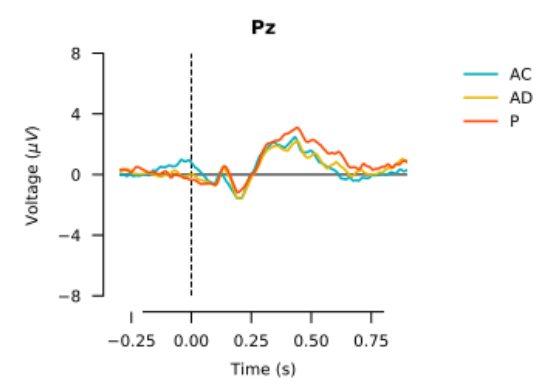
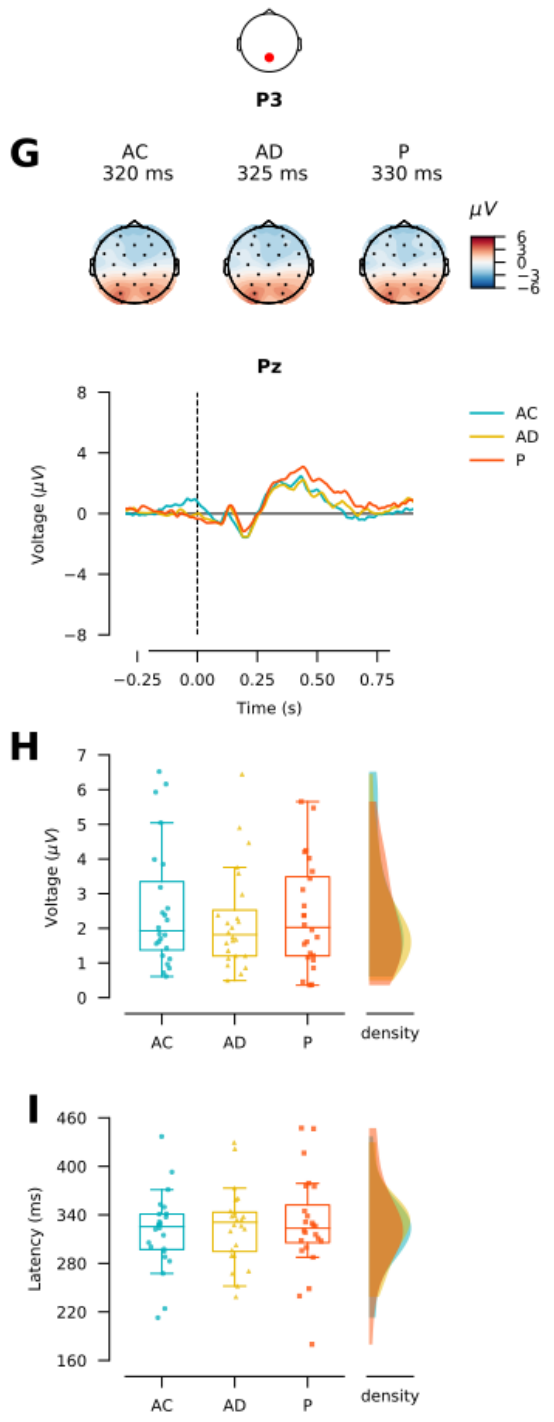

Figure 3. Agents' self-initiation modulates early visual and attentional ERPs indexes. A. Upper panel Topographical plots of the indicated times. Dots represent modeled electrode positions; red: positive voltage; blue: negative voltage; values in $\mu \mathrm{V}$. Bottom panel. ERP grand average ( $n=24$ subjects) evoked by stimuli presentation ( $t=0 \mathrm{~ms}$ ) in electrode $\mathrm{Oz}$, per conditions. Only correct trials are included. B. Left panel, box plot shows the peak-to-peak voltage $(\mu \mathrm{V})$ of N1 component, per condition. Right panel, density plot shows the distributions of peak-to-peak voltage of $\mathrm{N} 1$ per encoding condition. C. Similar to B, but for N1 latency (in ms). D. Similar to A, but for electrode Fz. E. Similar to B, but for P2 peak-to-peak amplitude. F. Similar to B, but for P2 latency G. Similar to A, but for electrode Pz. H. Similar to B, but for P3 peak-topeak amplitude. I. Similar to B, but for P3 latency. $\left({ }^{*}: p \leq 0.05 ;{ }^{* *}: p \leq 0.01 ;{ }^{* * *}: p \leq 0.0001\right)$. 


\section{Earlier attentional processing is a marker of a coupled active phenomenon}

Next, we explored whether the earlier deploying time of visual and/or attentional processing provides reliable markers of self-initiation of stimuli. To do so, we performed a conditional Classification Tree (CART model) that allowed us to test how reliably we can estimate the experimental condition of a trial from the latency of its associated P1 and P2 components. Since CART models are ideal for classifying or evaluating discrete state variables (Strobl et al. 2009), by using this method, it is assumed that initiating the trial in a coupled way would deploy a phenomenon presumably absent during decoupled or passive conditions. CART model results show that the latency of the P2 component better distinguishes between encoding conditions, using a cutoff of $175.293 \mathrm{~ms}(\mathrm{p}<0.001)$ (See Fig. 4A). No other ERP variable had a significant effect according to the model. Latencies below 175 ms corresponded mostly to the AC condition ( $\mathrm{AC}$ median $=169.92 \pm 10.14 \mathrm{~ms})$, while later P2 latencies tended to be found in AD (median = $184.81 \pm 10.2 \mathrm{~ms}$ ) and $\mathrm{P}$ conditions (median $=190.67 \pm 8.09 \mathrm{~ms}$ ). This result suggests that the attentional index's onset time is the variable that better distinguishes a coupled self-initiated encoding process from a decoupled or a passive one.

Since earlier P2 latency is the variable that better distinguishes the AC condition and that $\mathrm{AC}$ is also the condition that shows better accuracy (See Fig. 2B), we analyzed if P2 latency can explain the performance improvement by itself or if it is dependent on the task condition. When contrasted with accuracy, P2 latencies of the AC condition ( $\leq 175 \mathrm{~ms})$ tend to show higher accuracy values (Fig. 4B). On the other hand, P2 latencies later than 175 ms (mostly compound of $A D$ and $P$ conditions values) tend to show worst accuracy with later latency values (Fig. $4 \mathrm{~B}$ ). To test if this observed relation between P2 and accuracy is dependent on the task condition, we designed a Linear mixed model including accuracy as a dependent variable, P2 latency as a fixed effect, and encoding conditions as random effect variable. Results yield that there is no significant effect of P2 latency on accuracy when including task conditions as random effect variable $(\boldsymbol{\beta}=-$ 
$0.001 \pm 9 \mathrm{e}-04, \mathrm{t}=-1.259, \mathrm{p}=0.106)$. This result supports that it is not P2 latency by itself that mediates WM improvement.

In summary, the findings show that P2 latency is the variable that better distinguishes a coupled self-initiated encoding process from a decoupled or a passive one, and that it is not P2 latency by itself that mediates WM improvement but the precise nature of the coupled self-initiation condition.

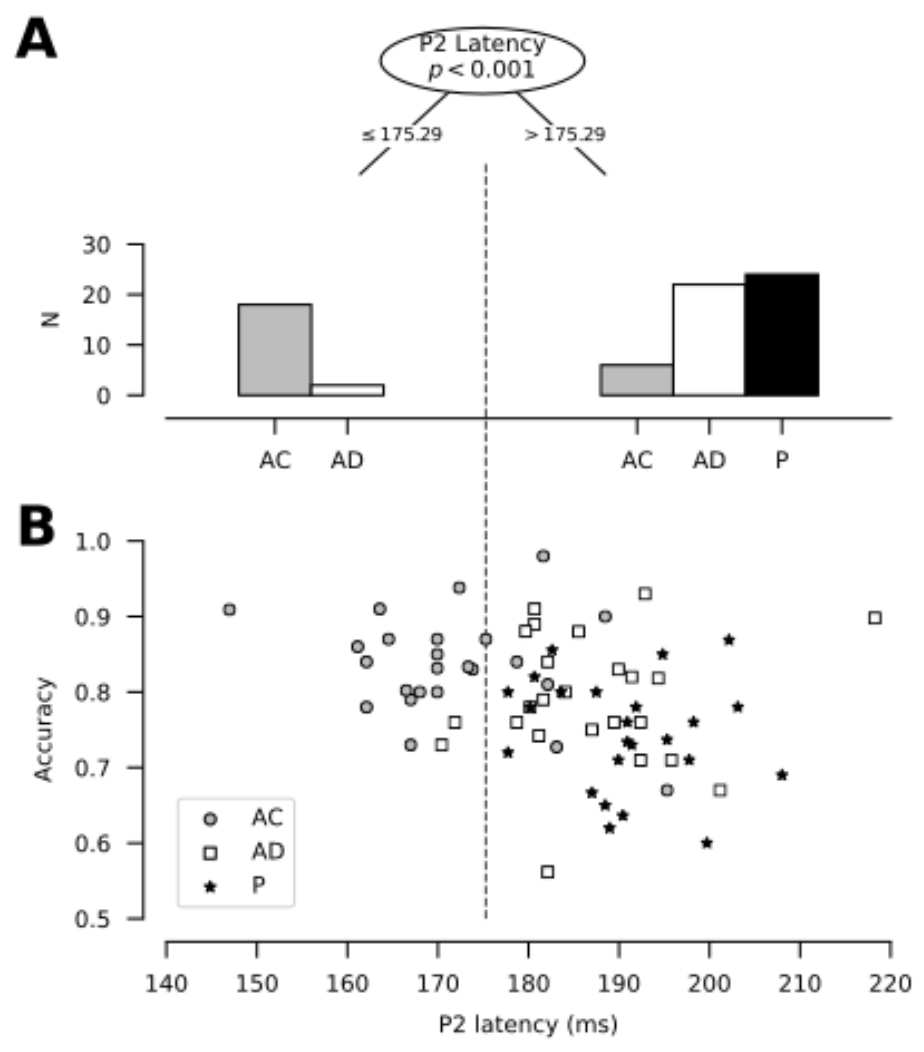

Figure 4. CART model of encoding conditions. A. Histograms of the number of participants per encoding condition (y-axis) attributed by the CART node based on P2 latency, with a split at $175.29 \mathrm{~ms}$ (top). The left histogram represents latencies lower than the split. Conversely, the right histogram represents latencies larger than the split. The number of cases per condition is equal to 24. B. Scatterplot of Accuracy (y-axis) as a function of the latency of the P2 component (x-axis), depicted by the condition $\left(\bullet=A C ; \square=A D ;{ }^{*}=P\right)$. The discontinuous vertical dashed line represents the split value of the CART model (175.293 ms). Each mark (whether circle, square or star) represents one participant ( $n=24$ per encoding condition). 


\section{DISCUSSION}

The current study assesses whether the agency modulates WM encoding through temporal modulation of visual and attentional processing. This was investigated by evaluating the influence of active self-initiation of the stimuli on behavior in a Sternberg working memory task, while concurrently measuring ERPs' encoding components widely used as indexes of visual (N1), attentional (P2) activity, and memory updating mechanisms (P3).

\section{Agency enhances WM performance and accelerates visual and attentional processing}

Our results show that actively initiating the stimuli's presentation leads to performance enhancement in a WM task. This finding is consistent with previous research stating that WM and motor systems share cognitive resources (Weigelt et al. 2009; Spiegel et al. 2013; Logan \& Fischman 2015; Buszard et al. 2016; Gündüz et al. 2017). The present data support that behavioral enhancement is not explained as differences in task difficulty since there is no distinction in the perceived confidence among the three task conditions. Alongside this, there is no statistically significant difference in reaction times (RT), which is classically modulated by task difficulty (Nickerson 1972; Pins \& Bonnet 1997; Schneider \& Anderson 2011). Our findings rather suggest a role of temporal predictability or motor systems modulation on cognition.

It has been widely proposed that motor actions can predict the timing (i.e., "when") of the stimulus onset (Saleh et al. 2010; Fujioka et al. 2012; Arnal \& Giraud 2012). Motor regions such as the primary sensorimotor cortex (SM1), supplementary motor area (SMA), and cerebellum have been proved to be sensitive to auditory regularities even when auditory stimuli are not under the focus of attention, suggesting a role of these cortices on the temporal prediction of stimuli (Fujioka et al. 2012). While temporal prediction mediated by both overt and covert movements tend to reduce sensation and its neural correlates (Blakemore et al. 1999; Voss et al. 2008; Wolpe et al. 2018; Klaffehn et al. 2019; but for a contradictory effect, see Rohenkohl et al. 2014), no evidence 
of such reduction in components' amplitude was found in the current work (See Fig. 3). On the contrary, our results show a temporal modulatory effect on visual processing, which is more in line with previous studies suggesting that motor-mediated temporal prediction facilitates sensorial processing (Ito et al. 2011; Devia, Montefusco-Siegmund, Egaña, Maldonado 2017; Schwarz et al. 2018). On the other hand, when temporal prediction interacts with both bottom-up and topdown attentional orientation mechanisms, time prediction boosts performance and its neural correlates (Kok et al. 2012; Marchant \& Driver 2013; Morillon et al. 2016; Kaiser \& Schütz-Bosbach 2018). Consistent with these reports, our findings show that time-locked motor-mediated triggering of the stimuli is associated with both attentional acceleration and performance improvement in a WM task.

Furthermore, our results suggest that, although coupled self-initiation of the stimuli is correlated to earlier sensory and attentional processes, the latter mechanism better distinguishes a coupled self-initiated encoding process from a decoupled or a passive one. It is noteworthy that no effects on the amplitudes of N1, P2, or P3 were found, suggesting that self-initiation effects are not based on modulating the number of neural populations required by the task but on influencing the time domain in which encoding is deployed. Altogether, coupled self-initiation of the stimuli enhances WM performance and engages a faster attentional state related to WM encoding, which seems to match the effects of temporal predictability previously reported. Our results support that this attentional state is not engaged during decoupled self-initiation.

If the agency's effect is solely based on temporal predictability, it should be suppressed under active-unpredictable situations. Our results show that this is not the case: decoupled actionstimuli triggering yields better accuracy and earlier latencies than passive stimuli onset. This result suggests that temporal predictability is not the only mechanism involved in the agency's WM effect. Embodied cognition theory states that subjects' bodies, particularly their motor systems, influence cognition (O'Regan \& Noë 2001; Varela et al. 2016). In agreement, sensory attenuation effect 
(i.e., the decreasing in neural sensory response due to self-initiation of a stimulus in a perceptual task) prevails even when compared to an externally generated tone, which is equally predictable in terms of time onset (Klaffehn et al. 2019), suggesting that sensory attenuation effect is not solely due to temporal predictability.

Moreover, decoupled movements modulate perception (Tomassini et al. 2015) and attention (Nittono 2007). There is also evidence suggesting that unrelated button pressing improves long-term memory encoding, whether the action is time-locked to stimulus onset or not (Yebra et al. 2019). Our results corroborate a parallel non-predictive modulation of motor systems over WM. This modulation is possibly related to the recruitment of catecholaminergic pathways, which will be discussed further.

\section{Agency does not modulate memory updating during WM encoding}

It was additionally hypothesized that agency could modulate the P3 component during WM encoding. P3 has been proposed as an index of the mental revision of WM stimuli (Polich 2007), and it seems to be related to posterior parietal cortex activity (Knight et al. 1989; Verleger et al. 1994). Classic WM studies have shown that the amplitude of P3 during encoding is an index of successful encoding, such that greater amplitude of P3 during encoding correlates with later successful retrieval (Karis et al. 1984; Fabiani et al. 1986). Our lack of effect on the P3 component implies that memory updating of stimuli in WM seems to be equal in both active and passive stimuli triggering. It should moreover be noted that the effect reported by Karis et al. (1984) and Fabiani et al. (1986) is dependent on task and rehearsal strategy used by the subjects, such that it is related to salient stimuli and non-elaborative memory rehearsal strategies (i.e., when subjects do not relate stimuli to retain them). These studies report that, even though elaborative rehearsal is associated with better accuracy, the relation between P3 amplitude and accuracy is not further evident when subjects use this strategy. 
In our work, subjects did not receive any special instruction about the rehearsal strategy, so it is possible that some of the participants could have used elaborative rehearsal strategies. As the strategy report was not requested, it is not feasible to corroborate if this can explain the lack of effect. On the other hand, our work did not manipulate the stimuli's saliency, being all consonants of the same size, contrast, and luminance. Future studies could explore the interaction between agency and bottom-up attentional mechanisms.

\section{The significance of the encoding stage of WM}

Notably, the proposal held in this work emphasizes that encoding processing is an equally relevant component of WM, since encoding implies WM mechanisms' initiation, using perception to memorize, in contrast to the user perception for purely discriminatory purposes. In this sense, as WM mechanisms engage during encoding, self-initiation modulates attentional mechanisms deployed during WM encoding, impacting WM's overall performance. The WMs' relationship with the encoding process as part of WM has been extensively explored in the auditory and verbal domain, such that the association between auditory perception-and its deficit-and verbal WM is well documented (e.g., Norrelgen et al. 2002; Wayne et al. 2016; Zhang et al. 2016; Guijo et al. 2018; Magimairaj \& Nagaraj 2018). This relationship appears to be less investigated in the visual modality (Cattaneo et al. 2011; Qian et al. 2017; Kosilo et al. 2017). It might be attributable due to the close connection between oral language and WM. One of the central and classic focuses of WM studies is its correlation to oral language, oral language development, and oral language disorders (e.g., Baddeley et al. 1998; Caplan \& Waters 1999; Montgomery 2003; Jonsdottir et al. 2005; Coelho et al. 2013; Vugs et al. 2013; Vugs et al. 2014; Fortunato-Tavares et al. 2015; Cogan et al. 2017). In this sense, the relationship between auditory deficits, cognitive effort, and language development appears to be more natural. On the contrary, and to the extent of our review, we did not find further research on visual signal degradation-either by experimental methods, such as 
luminance or contrast manipulations or by subjects' impairments, as low vision—on WM, which is relevant to assess due to the critical role of encoding within WM, as our findings have remarked it.

\section{Neural Model}

Based on the results of this study, we argue that the neural mechanisms underlying the time-coupled self-initiation effect on WM might be based on motor cortex modulation on perceptual and attentional neural systems. Motor cortices, such as M2 in rats or the premotor cortex in primates, are anatomically connected to the visual cortex (Attinger et al. 2017; Leinweber et al. 2017). Some theories propose that motor-sensory cortex projections may act as efference copies used by an internal forward model to predict the sensory consequence of the motor act (Miall \& Wolpert 1996; Wolpert \& Kawato 1998). A proposed role for these projections is the sense of agency, allowing the nervous system to discriminate when a sensory consequence is externally or self-generated (Poletti et al. 2017). Another role for motor-sensory cortex projections is to generate time predictions about the onset of sensory consequences, acting as a marker about when a sensory change will happen (Saleh et al. 2010; Arnal \& Giraud 2012). This would possibly occur through the modulation of local field potentials in the sensory cortex, as shown in the visual cortex (Ito et al. 2011). In our data, N100 lower latencies in time-coupled self-initiated conditions reflex early visual processing facilitation (Schroeder et al. 2010). This temporal modulation could be due to motor cortices activating motor-sensory cortex projections. This hypothesis should be tested in further studies, including methods for localizing the source of the electroencephalographic activity (which requires a greater number of recording channels) or magnetoencephalography.

Likewise, P2 earlier latencies in coupled self-initiated conditions can reflect the attentional processing's facilitation during WM encoding originated from the same motor cortices signaling. 
Our results suggest that this mechanism could operate in non-sensory cortex related to later attentional processing important to WM such as the dorsolateral prefrontal cortex (DLPFC) (Blumenfeld \& Ranganath 2006; D'Esposito \& Postle 2015) and posterior parietal cortex (PPC) (Todd \& Marois 2005; Curtis 2006; Berryhill \& Olson 2008). Motor cortices project to both DLPFC (Hasan et al. 2013) and PPC (Reep et al. 1994; Wilber et al. 2015), probably via the superior longitudinal fascicle connecting the supplementary motor cortex with the abovementioned cortices (Bozkurt et al. 2017). Therefore, movement-related activation of SMA might be signaling the income of a relevant stimulus to be maintained to DLPFC and PPC cortices as well. Consequently, this signal may facilitate the activation of the frontoparietal network underlying attention, such that this network might have a faster activation when it receives a motor signaling. Since corticocortical activations through direct anatomic projections occur within a few milliseconds (Leinweber et al. 2017), this effect should be visible shortly after the activation of the motor cortex. Hence, the temporality between the movement and the relevant stimuli presentation seems to be a relevant feature of the modulatory mechanism of the active self-initiation of the stimuli, as supported by previous studies (Blakemore et al. 1999; Morillon et al. 2014; Concha-Miranda et al. 2019) and our results. Notably, this appears not to affect the memory updating of WM stimuli, as our P3 results suggest, even though this component has possibly originated in the PPC cortex (Knight et al. 1989; Verleger et al. 1994).

Remarkably, the motor system can modulate cognition not only using direct corticocortical connections but also through modulatory effects of motor activation on dopamine, acetylcholine, and noradrenergic circuits. The dopamine system's importance in WM functioning has been extensively documented (Brozoski et al. 1979; Sawaguchi \& Goldman-Rakic 1991; Müller et al. 1998; Sawaguchi 2001). For instance, Müller et al. (1998) report a performance enhancement in a WM task when primates were administered with a D1/D2 dopamine agonist, possibly modulating DLPFC through the mesocortical pathway. On the other hand, dopamine depletion in monkeys' 
DLPFC induces deficits in visual WM tasks (Brozoski et al. 1979). Strikingly, the same authors demonstrate the reversion of the deficits when monkeys were administered with dopaminergic agonists such as L-dopa and apomorphine. Alongside this, it is known that the ventral tegmental area (VTA, which is related to the mesocortical circuit) is a signal target of motor cortices (Beier et al. 2015). Therefore, a voluntary movement could modulate dopaminergic activity, modulating DLPFC activity during active self-initiated WM encoding. Likewise, cholinergic neurons located in the basal prosencephalon show fast activity related to the current movement (Pinto et al. 2013; Eggermann et al. 2014; Nelson \& Mooney 2016). In turn, basal prosencephalon cholinergic neurons projects to DLPFC (Gielow \& Zaborszky 2017) and medial prefrontal cortex (Bloem et al. 2014) as well as to sensory cortices (Pinto et al. 2013; Eggermann et al. 2014; Nelson \& Mooney 2016). According to this view, a motor action could modulate movement-responsive basal prosencephalon cholinergic neurons, which in turn may modulate prefrontal and sensory cortices during active WM encoding. Finally, evidence shows locus coeruleus adrenergic neurons respond to voluntary movements (Kalwani et al. 2014; Bouret \& Richmond 2015). The adrenergic system has been correlated to enhancing long-term memory encoding (Cahill et al. 1994; Yebra et al. 2019). Since long-term memory is postulated as an important component of WM (Cowan 2005), it is possible that it could also participate in active self-initiated WM encoding. It is also known that basal ganglia seem to participate in sensorimotor, associative, and limbic information (Leisman \& Melillo 2013; Jahanshahi et al. 2015). As part of the associative circuit functioning, basal ganglia seem to have a supporting role in WM (Constantinidis \& Procyk 2004; Marvel et al. 2019). Previous studies report projections between the associative circuit of basal ganglia and the prefrontal cortex (Dum \& Strick 2009), specifically between DLPFC and internal globus pallidus-substantia nigra pars reticulata. Although basal ganglia are organized in three different topographically separated circuits, these circuits also overlap, allowing for the integration of associative, sensorimotor, and limbic signaling (Draganski et al. 2008). Thus, the activation of sensorimotor basal ganglia circuits 
due to a voluntary movement could influence associative basal ganglia circuits and possibly modulate WM.

Critically, the networks mentioned above require several synaptic relays to take place and thus could unravel more slowly than the modulatory effects of direct motor-sensory/associative cortices projections. Accordingly, the catecholamine system's recruitment and basal ganglia circuits by motor systems during active self-initiated encoding could explain the discrepancy between decoupled self-initiated encoding and passive encoding in both behavior and electrophysiology. On the other hand, fast direct cortico-cortical projections can explain the distinction between time-coupled and decoupled self-initiated encoding. While stimuli appearing coupled to the movement would be modulated by both fast (direct motor projections) and slow (activity related to catecholamines and basal ganglia circuits) modulatory networks, stimuli presented hundreds of milliseconds after the movement has been executed would be influenced by the slow response modulatory networks only. This would explain that both coupled and decoupled self-presentation lead to improved WM performance but that the effect is stronger in the coupled condition.

\section{Coda}

To conclude, the current findings show that agency, present during active self-initiation of the stimuli, modulates WM encoding processing through the influence of early sensory and attentional processes. Performance enhancement in coupled self-initiation is related to an earlier attentional state, which seems to be absent in passive and active decoupled self-initiated states. Nevertheless, processing facilitation of early sensory and attentional processes is also present in decoupled self-initiation compared to the stimuli's passive triggering. This suggests that active self-initiation, regardless of whether it is time-locked or not, engages a motor-mediated modulation on cognition in addition to the temporal predictive mechanism (the latter being absent in the 
decoupled self-initiated condition). Finally, our study also remarks that sensory and attentional processing during encoding is a crucial component of WM, emphasizing that WM does not merely maintain the mechanism of absent stimuli.

\section{Acknowledgments}

Special thanks are extended to Dr. Carolina Delgado, Dr. Marcela Peña, and Dr. Rómulo Fuentes for their valuable and constructive suggestions during the planning and development of this research work. We also extend our thanks to Mariana Mella for her assistance with the data acquisition, and to all the staff of Laboratorio de Neurosistemas.

\section{Funding}

This work was funded by the program of advanced human capital formation of the National Agency of Research and Development (Agencia Nacional de Investigación y Desarrollo, ANID former CONICYT-PFCHA; grant number 21150153 to R.L-N.) and Millennium Scientific Initiative (Iniciativa Científica Milenio, ICM; grant number P09-015-F to P.M.).

\section{Notes}

Conflict of Interest: None declared. 


\section{REFERENCES}

Arnal LH, Giraud AL. 2012. Cortical oscillations and sensory predictions. Trends Cogn Sci. 16(7):390-398.

Attinger A, Wang B, Keller GB. 2017. Visuomotor Coupling Shapes the Functional Development of Mouse Visual Cortex. Cell. 169(7):1291-1302.e14.

Baddeley A, Gathercole S, Papagno C. 1998. The Phonological Loop as a Language Learning Device. Psychol Rev. 105(1):158-173.

Bates D, Mächler M, Bolker BM, Walker SC. 2015. Fitting linear mixed-effects models using Ime4. J Stat Softw. 67(1):1-48.

Beier KT, Steinberg EE, Deloach KE, Xie S, Miyamichi K, Schwarz L, Gao XJ, Kremer EJ, Malenka RC, Luo L. 2015. Circuit Architecture of VTA Dopamine Neurons Revealed by Systematic Input-Output Mapping. Cell. 162(3):622-634.

Berryhill ME, Olson IR. 2008. Is the posterior parietal lobe involved in working memory retrieval?. Evidence from patients with bilateral parietal lobe damage. Neuropsychologia. 46(7):1775-1786.

Blakemore SJ, Frith CD, Wolpert DM. 1999. Spatio-temporal prediction modulates the perception of selfproduced stimuli. J Cogn Neurosci. 11(5):551-559.

Bloem B, Schoppink L, Rotaru DC, Faiz A, Hendriks P, Mansvelder HD, van de Berg WDJ, Wouterlood FG. 2014. Topographic mapping between basal forebrain cholinergic neurons and the medial prefrontal cortex in mice. J Neurosci.34(49):16234-16246.

Blumenfeld RS, Ranganath C. 2006. Dorsolateral prefrontal cortex promotes long-term memory formation through its role in working memory organization. J Neurosci. 26(3):916-925.

Bouret S, Richmond XJ. 2015. Sensitivity of locus ceruleus neurons to reward value for goal-directed actions. J Neurosci. 35(9):4005-4014.

Bozkurt B, Yagmurlu K, Middlebrooks EH, Cayci Z, Cevik OM, Karadag A, Moen S, Tanriover N, Grande AW. 2017. Fiber connections of the supplementary motor area revisited: Methodology of fiber dissection, DTI, and three-dimensional documentation. J Vis Exp. 2017(123).

Brozoski TJ, Brown RM, Rosvold HE, Goldman PS. 1979. Cognitive deficit caused by regional depletion of dopamine. Science. 205:929-932. 
Buszard T, Farrow D, Zhu FF, Masters RSW. 2016. The relationship between working memory capacity and cortical activity during performance of a novel motor task. Psychol Sport Exerc. 22:247-254.

Cahill L, Prins B, Weber M, McGaugh JL. 1994. $\beta$-Adrenergic activation and memory for emotional events. Nature. 371(6499):702-704.

Caplan D, Waters GS. 1999. Verbal working memory and sentence comprehension. Behav Brain Sci. 22(1):77126.

Cattaneo Z, Pisoni A, Papagno C, Silvanto J. 2011. Modulation of visual cortical excitability by working memory: Effect of luminance contrast of mental imagery. Front Psychol. 2(FEB):1-9.

Clark VP, Fan S, Hillyard SA. 1995. Identification of early VEP generators by retinotopic analyses. Hum Brain Mapp. 2:170-187.

Coelho S, Albuquerque CP, Simões MR. 2013. Specific language impairment: A neuropsychological characterization. Paideia. 23(54):31-41.

Cogan GB, lyer A, Melloni L, Thesen T, Friedman D, Doyle W, Devinsky O, Pesaran B. 2017. Manipulating stored phonological input during verbal working memory. Nat Neurosci. 20(2):279-286.

Concha-Miranda M, Ríos J, Bou J, Valdes JL, Maldonado PE. 2019. Timing is of the essence: improvement in perception during active sensing. Front Behav Neurosci. 13.

Constantinidis C, Procyk E. 2004. The primate working memory networks. Cogn Affect Behav Neurosci. $4(4): 444-465$.

Cowan N. 2005. Working Memory Capacity. Psychology Press.

Curtis CE. 2006. Prefrontal and parietal contributions to spatial working memory. Neuroscience. 139(1):173-180

D’Esposito M, Postle BR. 2015. The cognitive neuroscience of working memory. Annu Rev Psychol. 66:115142.

Devia C, Montefusco-Siegmund R, Egaña J, Maldonado P. 2017. Precise timing of sensory modulations coupled to eye movements during active vision. bioRxiv.:1-24.

Draganski B, Kherif F, Klöppel S, Cook PA, Alexander DC, Parker GJM, Deichmann R, Ashburner J, Frackowiak RSJ. 2008. Evidence for segregated and integrative connectivity patterns in the human basal ganglia. $J$ Neurosci. 28(28):7143-7152. 
Dum R, Strick P. 2009. Basal Ganglia and Cerebellar Circuits with the Cerebral Cortex. In: Gazzaniga M, editor. Cogn Neurosci. Cambridge, MA: MIT Press; p. 553-564.

Dunn BR, Dunn DA, Languis M, Andrews D. 1998. The relation of ERP components to complex memory processing. Brain Cogn. 36(3):355-376.

Eggermann E, Kremer Y, Crochet S, Petersen CCH. 2014. Cholinergic Signals in Mouse Barrel Cortex during Active Whisker Sensing. Cell Rep. 9(5):1654-1660.

Fabiani M, Karis D, Donchin E. 1986. P300 and Recall in an Incidental Memory Paradigm. Psychophysiology. 23(3):298-308.

Fortunato-Tavares T, Andrade CRF, Befi-Lopes D, Limongi SO, Fernandes FDM, Schwartz RG. 2015. Syntactic comprehension and working memory in children with specific language impairment, autism or Down syndrome. Clin Linguist Phonetics. 29(7):499-522.

Fox J, Weisberg S. 2014. An R Companion to Applied Regression: Appendices. Robust Regres R. (December):1-17.

Fujioka T, Trainor LJ, Large EW, Ross B. 2012. Internalized timing of isochronous sounds is represented in neuromagnetic beta oscillations. J Neurosci. 32(5):1791-1802.

Gielow MR, Zaborszky L. 2017. The Input-Output Relationship of the Cholinergic Basal Forebrain. Cell Rep. 18(7):1817-1830.

Gramfort A. 2013. MEG and EEG data analysis with MNE-Python. Front Neurosci. 7(7 DEC):267.

Green CS, Bavelier D. 2003. Action video game modifies visual selective attention. Nature. 423(6939):534-537.

Guijo LM, Horiuti MB, Nardez TMB, Cardoso ACV. 2018. Listening effort and working memory capacity in hearing impaired individuals: an integrative literature review. Rev CEFAC. 20(6):798-807.

Gündüz R, Schack T, Koester D. 2017. Movement interferes with visuospatial working memory during the encoding: An ERP study. Front Psychol. 8(MAY):1-16.

Hasan A, Galea JM, Casula EP, Falkai P, Bestmann S, Rothwell JC. 2013. Muscle and timing-specific functional connectivity between the dorsolateral prefrontal cortex and the primary motor cortex. J Cogn Neurosci. 25(4):558-570.

Holgado D, Zabala M, Sanabria D. 2019. No evidence of the effect of cognitive load on self-paced cycling performance. PLoS One . 14(5):e0217825. 
Hothorn T, Hornik K, Strobl C, Zeileis A. 2015. party: A Laboratory for Recursive Partytioning. R Packag version 09-0.

Hothorn T, Hornik K, Van De Wiel MA, Zeileis A. 2006. A lego system for conditional inference. Am Stat. 60(3):257-263.

Hothorn T, Hornik K, Zeileis A. 2006. Unbiased recursive partitioning: A conditional inference framework. J Comput Graph Stat. 15(3):651-674.

Ito J, Maldonado P, Singer W, Grün S. 2011. Saccade-related modulations of neuronal excitability support synchrony of visually elicited spikes. Cereb Cortex. 21(11):2482-2497.

Jahanshahi M, Obeso I, Rothwell JC, Obeso JA. 2015. A fronto-striato-subthalamic-pallidal network for goaldirected and habitual inhibition. Nat Rev Neurosci. 16(12):719-732.

Jonsdottir S, Bouma A, Sergeant JA, Scherder EJA. 2005. The impact of specific language impairment on working memory in children with ADHD combined subtype. Arch Clin Neuropsychol. 20(4):443-456.

Jurcak V, Tsuzuki D, Dan I. 2007. 10/20, 10/10, and 10/5 systems revisited: Their validity as relative headsurface-based positioning systems. Neuroimage. 34(4):1600-1611.

Kaiser J, Schütz-Bosbach S. 2018. Sensory attenuation of self-produced signals does not rely on self-specific motor predictions. Eur J Neurosci. 47(11):1303-1310.

Kalwani RM, Joshi S, Gold JI. 2014. Phasic Activation of Individual Neurons in the Locus Ceruleus/Subceruleus Complex of Monkeys Reflects Rewarded Decisions to Go But Not Stop. J Neurosci. 34(41):13656-13669.

Karis D, Fabiani M, Donchin E. 1984. "P300” and memory: Individual differences in the von Restorff effect. Cogn Psychol. 16(2):177-216.

Klaffehn AL, Baess P, Kunde W, Pfister R. 2019. Sensory attenuation prevails when controlling for temporal predictability of self- and externally generated tones. Neuropsychologia. 132:107145.

Knight RT, Scabini D, Woods DL, Clayworth CC. 1989. Contributions of Temporal-Parietal Junction to human auditory P3. Brain Res. 502(1):109-116.

Kok P, Rahnev D, Jehee JFM, Lau HC, De Lange FP. 2012. Attention reverses the effect of prediction in silencing sensory signals. Cereb Cortex. 22(9):2197-2206.

Kolb H. 1995. Facts and Figures Concerning the Human Retina. Webvision Organ Retin Vis Syst. (1):1-11. 
Kosilo M, Martinovic, Laxhman, Lisshammar J, Barbur, Haenschel C. 2017. Contribution of Luminance to Visual Working Memory in Schizophrenia.

Kumar N, Manjaly JA, Sunny MM. 2015. The relationship between action-effect monitoring and attention capture. J Exp Psychol Gen. 144(1):18-23.

Lawrence MA. 2016. ez: Easy analysis and visualization of factorial experiments.

Leinweber M, Ward DR, Sobczak JM, Attinger A, Keller GB. 2017. Erratum: A Sensorimotor Circuit in Mouse Cortex for Visual Flow Predictions Neuron. 96(5):1204.

Leisman G, Melillo R. 2013. The basal ganglia: Motor and cognitive relationships in a clinical neurobehavioral context. Rev Neurosci. 24(1):9-25.

Logan SW, Fischman MG. 2015. The death of recency: Relationship between end-state comfort and serial position effects in serial recall: Logan and Fischman (2011) revisited. Hum Mov Sci. 44:11-21.

Luck SJ, Heinze HJ, Mangun GR, Hillyard SA. 1990. Visual event-related potentials index focused attention within bilateral stimulus arrays. II. Functional dissociation of P1 and N1 components. Electroencephalogr Clin Neurophysiol. 75(6):528-542.

Magimairaj BM, Nagaraj NK. 2018. Working memory and auditory processing in school-age children. Lang Speech Hear Serv Sch. 49(3):409-423.

Marchant JL, Driver J. 2013. Visual and audiovisual effects of isochronous timing on visual perception and brain activity. Cereb Cortex. 23(6):1290-1298.

Marvel CL, Morgan OP, Kronemer SI. 2019. How the motor system integrates with working memory. Neurosci Biobehav Rev. 102(April):184-194.

McFarland JM, Bondy AG, Saunders RC, Cumming BG, Butts DA. 2015. Saccadic modulation of stimulus processing in primary visual cortex. Nat Commun. 6:1-14.

McMillan KM, Laird AR, Witt ST, Meyerand ME. 2007. Self-paced working memory: Validation of verbal variations of the n-back paradigm. Brain Res. 1139(1):133-142.

Miall RC, Wolpert DM. 1996. Forward models for physiological motor control. Neural Networks.

Missonnier P, Deiber MP, Gold G, Herrmann FR, Millet P, Michon A, Fazio-Costa L, Ibañez V, Giannakopoulos P. 2007. Working memory load-related electroencephalographic parameters can differentiate progressive from stable mild cognitive impairment. Neuroscience. 150(2):346-356. 
Missonnier P, Gold G, Leonards U, Costa-Fazio L, Michel JP, Ibáñez V, Giannakopoulos P. 2004. Aging and working memory: Early deficits in EEG activation of posterior cortical areas. J Neural Transm. 111(9):11411154.

Montgomery JW. 2003. Working memory and comprehension in children with specific language impairment: What we know so far. J Commun Disord. 36(3):221-231.

Morillon B, Schroeder CE, Wyart V. 2014. Motor contributions to the temporal precision of auditory attention. Nat Commun. 5:1-9.

Morillon B, Schroeder CE, Wyart V, Luc X, Arnal H. 2016. Brief Communications.

Moscatelli A, Mezzetti M, Lacquaniti F. 2012. Modeling psychophysical data at the population-level: The generalized linear mixed model. J Vis. 12(11):1-17.

Müller MM, Teder-Sälejärvi W, Hillyard SA. 1998. The time course of cortical facilitation during cued shifts of spatial attention. Nat Neurosci. 1(7):631-634.

Nasreddine ZS, Phillips NA, Bédirian V, Charbonneau S, Whitehead V, Collin I, Cummings JL, Chertkow H. 2005. The Montreal Cognitive Assessment, MoCA: A brief screening tool for mild cognitive impairment. J Am Geriatr Soc. 53(4):695-699.

Natale E, Marzi CA, Girelli M, Pavone EF, Pollmann S. 2006. ERP and fMRI correlates of endogenous and exogenous focusing of visual-spatial attention. Eur J Neurosci. 23(9):2511-2521.

Nelson A, Mooney R. 2016. The Basal Forebrain and Motor Cortex Provide Convergent yet Distinct MovementRelated Inputs to the Auditory Cortex. Neuron. 90(3):635-648.

Nickerson RS. 1972. Binary-classification reaction time: A review of some studies of human informationprocessing capabilities. Psychon Monogr Suppl. 4(17):275-318.

Nittono H. 2004. The action-perception paradigm: A new perspective in cognitive neuroscience. Int Congr Ser. 1270(C):26-31.

Nittono H. 2006. Voluntary stimulus production enhances deviance processing in the brain. Int J Psychophysiol. 59(1):15-21.

Nittono H. 2007. Event-related brain potentials corroborate subjectively optimal delay in computer response to a user's action. Lect Notes Comput Sci (including Subser Lect Notes Artif Intell Lect Notes Bioinformatics). 4562 LNAI:575-581. 
Nittono H, Hamada A, Hori T. 2003a. Brain Potentials after Clicking a Mouse: A New Psychophysiological Approach to Human-Computer Interaction. Hum Factors. 45(4):591-599.

Nittono H, Hamada A, Hori T. 2003b. Brain Potentials after Clicking a Mouse: A New Psychophysiological Approach to Human-Computer Interaction. Hum Factors. 45(4):591-599.

Norrelgen F, Lacerda F, Forssberg H. 2002. Temporal resolution of auditory perception and verbal working memory in 15 children with language impairment. J Learn Disabil. 35(6):539-545.

O’Regan JK, Noë A. 2001. A sensorimotor account of vision and visual consciousness. Behav Brain Sci. 24(5):939-973.

Pins D, Bonnet C. 1997. Reaction times reveal the contribution of the different receptor components in luminance perception. Psychon Bull Rev. 4(3):359-366.

Pinto L, Goard MJ, Estandian D, Xu M, Kwan AC, Lee SH, Harrison TC, Feng G, Dan Y. 2013. Fast modulation of visual perception by basal forebrain cholinergic neurons. Nat Neurosci. 16(12):1857-1863.

Poletti M, Gebhardt E, Raballo A. 2017. Corollary Discharge, Self-agency, and the Neurodevelopment of the Psychotic Mind. JAMA Psychiatry. 74(11):1169-1170.

Polich J. 2007. Updating P300: An integrative theory of P3a and P3b. Clin Neurophysiol. 118(10):2128-2148.

Postle BR. 2006. Working memory as an emergent property of the mind and brain. Neuroscience. 139(1):2338.

Proksch J, Bavelier D. 2002. Changes in the spatial distribution of visual attention after early deafness. J Cogn Neurosci. 14(5):687-701.

Qian J, Li J, Wang K, Liu S, Lei Q. 2017. Evidence for the effect of depth on visual working memory. Sci Rep. $7(1): 1-10$.

Reep RL, Chandler HC, King V, Corwin J V. 1994. Rat posterior parietal cortex: topography of corticocortical and thalamic connections. Exp Brain Res. 100(1):67-84.

Rohenkohl G, Cravo AM, Wyart V, Anna C. 2014. Temporal expectation improves the quality of sensory information. 32(24):8424-8428.

Di Russo F, Martínez A, Sereno MI, Pitzalis S, Hillyard SA. 2002. Cortical sources of the early components of the visual evoked potential. Hum Brain Mapp. 15(2):95-111. 
Saleh M, Reimer J, Penn R, Ojakangas CL, Hatsopoulos NG. 2010. Fast and Slow Oscillations in Human Primary Motor Cortex Predict Oncoming Behaviorally Relevant Cues. Neuron. 65(4):461-471.

Sawaguchi T. 2001. The effects of dopamine and its antagonists on directional delay-period activity of prefrontal neurons in monkeys during an oculomotor delayed-response task. Neurosci Res. 41(2):115-128.

Sawaguchi T, Goldman-Rakic PS. 1991. D1 dopamine receptors in prefrontal cortex: Involvement in working memory. Science. 4996(251):947-950.

Schneider DW, Anderson JR. 2011. A memory-based model of Hick's law. Cogn Psychol. 62(3):193-222.

Schroeder CE, Wilson DA, Radman T, Scharfman H, Lakatos P. 2010. Dynamics of Active Sensing and perceptual selection. Curr Opin Neurobiol. 20(2):172-176.

Schwarz KA, Pfister R, Kluge M, Weller L, Kunde W. 2018. Do we see it or not? Sensory attenuation in the visual domain. J Exp Psychol Gen. 147(3):418-430.

Sergent C, Baillet S, Dehaene S. 2005. Timing of the brain events underlying access to consciousness during the attentional blink. Nat Neurosci. 8(10):1391-1400.

Spiegel MA, Koester D, Schack T. 2013. The functional role of working memory in the (Re-)planning and execution of grasping movements. J Exp Psychol Hum Percept Perform. 39(5):1326-1339.

Stenner MP, Bauer M, Haggard P, Heinze HJ, Dolan R. Enhanced alpha-oscillations in visual cortex during anticipation of self-generated visual stimulation. J Cogn Neurosci. 2014 Nov;26(11):2540-51.

Stenner MP, Bauer M, Heinze HJ, Haggard P, Dolan RJ. 2015. Parallel processing streams for motor output and sensory prediction during action preparation. J Neurophysiol. 113(6):1752-1762.

Sternberg S. 1966. High-speed scanning in human memory. Science. 153(3736):652-654.

Strobl C, Malley J, Tutz G. 2009. An Introduction to Recursive Partitioning: Rationale, Application, and Characteristics of Classification and Regression Trees, Bagging, and Random Forests. Psychol Methods. 14(4):323-348.

Teng C, Kravitz DJ. 2019. Visual working memory directly alters perception. Nat Hum Behav. 3(8):827-836.

Todd JJ, Marois R. 2005. Posterior parietal cortex activity predicts individual differences in visual short-term memory capacity. Cogn Affect Behav Neurosci. 5(2):144-155.

Tomassini A, Spinelli D, Jacono M, Sandini G, Morrone MC. 2015. Rhythmic oscillations of visual contrast sensitivity synchronized with action. J Neurosci. 35(18):7019-7029. 
Varela FJ, Thompson E, Rosch E, Kabat-Zinn J. 2016. The embodied mind: Cognitive science and human experience. Embodied Mind Cogn Sci Hum Exp.:1-322.

Verleger R, Heide W, Butt C, Kömpf D. 1994. Reduction of P3b in patients with temporo-parietal lesions. Cogn Brain Res. 2(2):103-116.

Vogel EK, Luck SJ. 2000. The visual N1 component as an index of a discrimination process. Psychophysiology. 37(2):190-203.

Voss M, Ingram JN, Wolpert DM, Haggard P. 2008. Mere expectation to move causes attenuation of sensory signals. PLoS One. 3(8).

Vugs B, Cuperus J, Hendriks M, Verhoeven L. 2013. Visuospatial working memory in specific language impairment: A meta-analysis. Res Dev Disabil. 34(9):2586-2597.

Vugs B, Hendriks M, Cuperus J, Verhoeven L. 2014. Working memory performance and executive function behaviors in young children with SLI. Res Dev Disabil. 35(1):62-74.

Walsh E, Haggard P. The effects of acoustic startle on sensorimotor attenuation prior to movement. 2008 Exp Brain Res. Aug;189(3):279-88.

Waszak F, Herwig A. 2007. Effect anticipation modulates deviance processing in the brain. Brain Res. 1183(1):74-82.

Wayne R V., Hamilton C, Huyck JJ, Johnsrude IS. 2016. Working memory training and speech in noise comprehension in older adults. Front Aging Neurosci. 8(MAR):1-15.

Weigelt M, Rosenbaum DA, Huelshorst S, Schack T. 2009. Moving and memorizing: Motor planning modulates the recency effect in serial and free recall. Acta Psychol (Amst). 132(1):68-79.

Wen W. 2019. Does delay in feedback diminish sense of agency? A review. Conscious Cogn. 73:102759.

Wilber AA, Clark BJ, Demecha AJ, Mesina L, Vos JM, McNaughton BL. 2015. Cortical connectivity maps reveal anatomically distinct areas in the parietal cortex of the rat. Front Neural Circuits. 8(JAN):146.

Wolpe N, Zhang J, Nombela C, Ingram JN, Wolpert DM, Rowe JB, Tyler LK, Brayne C, Bullmore ET, Calder AC, et al. 2018. Sensory attenuation in Parkinson's disease is related to disease severity and dopamine dose. Sci Rep. 8(1):1-10.

Wolpert DM, Kawato M. 1998. Multiple paired forward and inverse models for motor control. Neural Networks. 
Yebra M, Galarza-Vallejo A, Soto-Leon V, Gonzalez-Rosa JJ, de Berker AO, Bestmann S, Oliviero A, Kroes MCW, Strange BA. 2019. Action boosts episodic memory encoding in humans via engagement of a noradrenergic system. Nat Commun. 10(1):1-12.

Zhang X, Yan W, Wang W, Fan H, Hou R, Chen Y, Chen Z, Ge C, Duan S, Compte A, Li CT. 2019. Active information maintenance in working memory by a sensory cortex. Elife. 8 .

Zhang YX, Moore DR, Guiraud J, Molloy K, Yan TT, Amitay S. 2016. Auditory discrimination learning: Role of working memory. PLoS One. 11(1):1-18. 